\title{
Expression of the PXR gene in various types of cancer and drug resistance (Review)
}

\author{
ENQI QIAO ${ }^{1}$, MINGHUA JI $^{1}$, JIANZHONG WU ${ }^{1}$, RONG MA $^{1}$, XIAOHUA ZHANG $^{2}$, \\ YUEJUN HE ${ }^{3}$, QUANBIN ZHA ${ }^{3}$, XUE SONG $^{1}$, LI-WEI ZHU ${ }^{1}$ and JINHAI TANG ${ }^{1}$ \\ ${ }^{1}$ Department of General Surgery, Jiangsu Cancer Hospital, Affiliated to Nanjing Medical University, Nanjing 210009; \\ ${ }^{2}$ Department of Oncology, The First Affiliated Hospital with Nanjing Medical University, Nanjing 210009; \\ ${ }^{3}$ Department of General Surgery, Xuzhou Medical College, Xuzhou 221000, P.R. China
}

Received October 10, 2012; Accepted January 2, 2013

DOI: $10.3892 / \mathrm{ol} .2013 .1149$

\begin{abstract}
Pregnane $X$ receptor (PXR) is a member of the nuclear receptor superfamily of ligand-regulated transcription factors. PXR is a key xenobiotic receptor that regulates the expression of genes implicated in drug metabolism, detoxification and clearance, including drug metabolizing enzymes and transporters, suggesting that it is significant in the drug resistance of cancer cells. PXR is expressed in a wide range of tissues in the human body. Studies have demonstrated that PXR is expressed in a variety of tumor types, correlating not only with drug resistance but also with the cell proliferation, apoptosis and prognosis of cancer. The purpose of the present review is to provide a comprehensive review of PXR and its potential roles in multidrug resistance and the biological characteristics of PXR-positive tumors.
\end{abstract}

\section{Contents}

1. Pregnane $X$ receptor

2. Correlation between the expression of PXR in various types of cancer and drug resistance

3. PXR and tumor cell apoptosis

4. Preventing drug resistance by regulating PXR

5. Conclusions and prospects

\section{Pregnane $X$ receptor}

Nomenclature. Pregnane X receptor (PXR; NR1I2) belongs to the NR1I subfamily of nuclear receptors (NRs). PXR was

Correspondence to: Professor Jinhai Tang, Department of General Surgery, Jiangsu Cancer Hospital, Affiliated to Nanjing Medical University, 42 bai zi ting, Nanjing 210009, Jiangsu, P.R. China E-mail: tangjinhaidoctor@sina.cn

Key words: pregnane $\mathrm{X}$ receptor, multidrug resistance, cancer, tumor cell apoptosis discovered by several groups in 1998 (1-4) and is alternatively referred to as the steroid and xenobiotic receptor (PXR) and pregnane-activated receptor (PAR), also known as SXR or hPXR in humans.

Structure of PXR. PXR contains the highly conserved DNA-binding domain (DBD), a characteristic structure of NRs. The far N-terminus is a short activation function-1 (AF-1) region which permits the regulation of receptor action in a ligand-independent manner. The structure of the PXR DBD is highly similar to that of the retinoid X receptor $\alpha(\mathrm{RXRa}) \mathrm{DBD}$, which is a double zinc-finger motif that contacts DNA in a sequence-specific fashion. The response elements include direct repeats with 3 to 5 bases separating the DBD binding sites (DR-3, DR-4 and DR-5) and inverted repeats (with the beginning of each sequence in proximity) separated by 6 or 8 bases (ER- 6 and ER-8, respectively) $(1,4,5)$. The two most important PXR target genes, multidrug resistance protein 1 (MDR1) and cytochrome P450 3A4 (CYP3A4), contain DR-4/ER-6 (6) and DR-3/ER-6 (4,7) in their promoter regions, respectively. The PXR DBD is also reported to contain a bipartite nuclear localization sequence (8). The DBD is linked to the ligandbinding domain (LBD) in PXR by a hinge region which is considerably shorter than that observed in the majority of NRs. The LBD contains the ligand-dependent activation function 2 region and the ligand-binding pocket. It is possible for the LBD of PXR to heterodimerize with the LBD of RXRa, similar to the known structures of other NR LBDs with the RXRa LBDs (9). Conformational changes upon ligand binding in AF-2, which are responsible for the recruitment of coregulator proteins, lead to changes in the transcription of target genes (Fig. 1) (10-12).

The three-dimensional structure of the LBD in PXR reveals that it has a large, spherical ligand-binding cavity which enables it to interact with a wide range of hydrophobic chemicals. Structural flexibility appears to be extremely important to allow PXR to conform to a series of ligands that differ in size and shape. Therefore, unlike other NRs which interact selectively with their physiological ligands, PXR serves as a generalized sensor of hydrophobic substances (Fig. 2). 
Function of PXR. Unlike the majority of NRs, the list of genes which are regulated by PXR is growing rapidly, including not only the systems associated with drug and xenobiotic metabolism but also those central to cholesterol and bile acid metabolism and excretion.

PXR has been shown to regulate the expression of genes involved in the oxidation (phase I), conjugation (phase II) and transport (phase III) of xenobiotics, which promote the metabolism, elimination and detoxification of chemotherapeutic agents. Phase I drug metabolizing enzymes (DMEs), regulated by PXR, include a number of CYPs, carboxylesterases, alcohol and aldehyde dehydrogenases and enzymes involved in heme production and the P450 reaction cycle $(13,14)$. PXR is a significant regulator of the xenobiotic-responsive expression of CYP3A genes. Accordingly, PXR is highly expressed in the human liver and intestine, where CYP3A is abundant and capable of metabolizing a broad range of structurally diverse xenobiotics $(15,16)$. A large number of compounds that induce CYP3A expression are also PXR activators (17). Phase II DMEs which are regulated by PXR facilitate the excretion of phase I biotransformed xenobiotics, including glucuronyl transferases (UGTs), sulfotransferases (SULTs) and glutathione transferases (GSTs) (18). UGT-catalyzed glucuronidation reactions are vital for the clearance of bilirubin and xenobiotics. A number of UGTs, including UGT1A1, UGT1A3, UGT1A4, UGT1A6 and UGT1A9, have been identified as PXR targets (19-24). Numerous efflux transporters, including $A B C$ drug efflux transporters, breast cancer resistance protein (BCRP), multidrug resistance-associated proteins (MRPs) and P-glycoprotein (P-gp) are also regulated by PXR $(6,15,16,25,26)$.

PXR coordinately regulates a large proportion of genes and proteins in the liver, intestine and other organs that are involved in all aspects of the detoxification and elimination of xenobiotics and drugs.

PXR is unique in the NR superfamily since it responds promiscuously to a wide range of chemically-distinct ligands from 232 to $>800 \mathrm{Da}(1-3,25,27)$. The induction of CYP3A4 expression represents the basis for an important class of drug-drug interactions in which one drug accelerates the metabolism of other drugs. It is evident that the majority of the prescription drugs which induce the expression of CYP3A4 do so by activating PXR. Furthermore, PXR has also been associated with the interaction between the herbal remedy St. John's wort (SJW) and prescription drugs (28). The knowledge that PXR is the molecular basis for common drug-drug interactions should contribute to the development of safer medicines.

Numerous studies have demonstrated new and mostly unexpected roles for PXR in regulating inflammation, bone homeostasis, energy homeostasis, lipid homeostasis and cancer.

Distribution of PXR. The expression of PXR is widespread in the tissues of humans and rodents. PXR is highly expressed in the liver, small intestine and colon in humans, rabbits, rats and mice (1-4,27-30). Notably, these are also the same tissues where the $C Y P 3 A$ genes are most highly induced and expressed. In rodents, PXR mRNA has been detected in the kidney (31), brain (32), lung (33) stomach, ovary, placenta $(34,35)$, immune cells (36), peripheral mononuclear blood cells $(37,38)$, heart, bone marrow and spinal cord (39). PXR is expressed not only

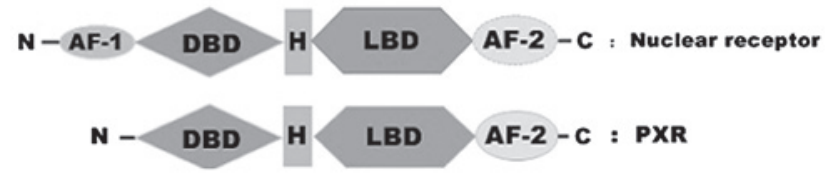

Figure 1. Schematic comparison of the domain structures of a typical nuclear receptor and PXR. AF-1, activation function-1; DBD, DNA-binding domain; $\mathrm{H}$, hinge region; LBD, ligand-binding domain; AF-2, transactivation function-2; PXR, pregnane $\mathrm{X}$ receptor.

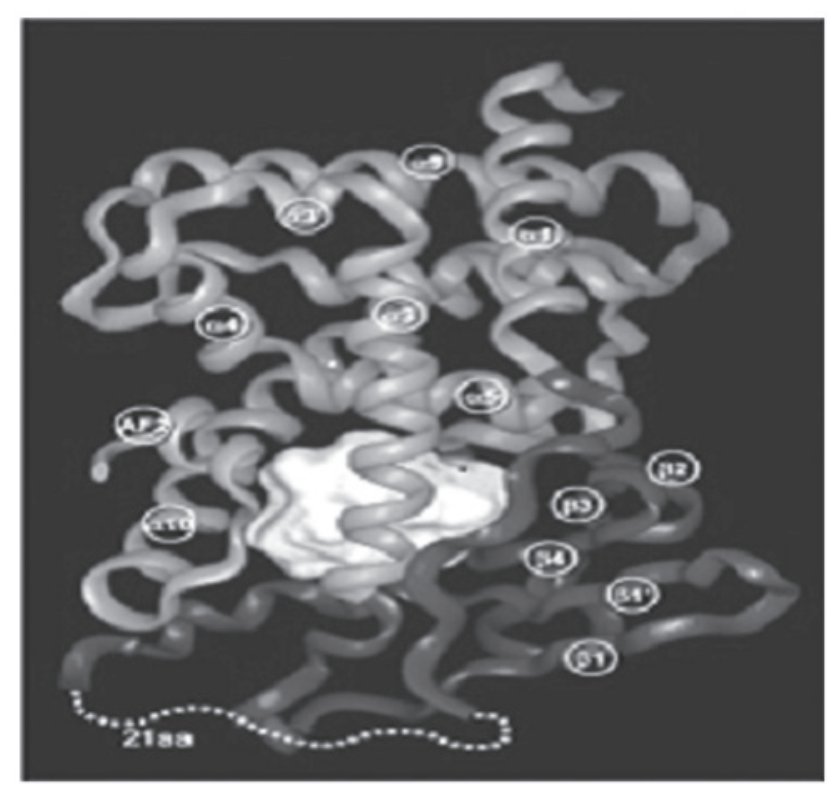

Figure 2. Structure of the PXR LBD. The three-dimensional structure of the human PXR LBD is presented as a ribbon diagram. The $\alpha$-helices, $\beta$-sheets and the AF-2 helix are indicated. The helix 1-helix 3 insert, which is unique to PXR among the NRs, is indicated in dark grey. The large solvent-accessible ligand-binding pocket is outlined in white. $\mathrm{PXR}$, pregnane $\mathrm{X}$ receptor; LBD, ligand-binding domain; AF-2, transactivation function-2; NR, nuclear receptor. This figure is taken from ref. 84.

in normal tissues, but also in numerous types of human cancer, including breast $(40,41)$, osteosarcoma (42), colon (43), endometrial $(44,45)$, ovarian $(46)$, prostate $(47)$ and esophageal (48) cancers. Most significantly, the expression levels of PXR in these cancer tissues are usually higher than in non-neoplastic tissues.

\section{Correlation between the expression of PXR in various types of cancer and drug resistance}

PXR and colon cancer. Of all types of cancer, studies of the correlation between PXR and colon cancer are the most common.

Pfrunder et al (49) noted that hPXR mRNA was highly expressed in three colon cancer cell lines, Caco-2 parental, Caco-2 TC-7 (TC-7) and LS180. Jiang et al (50) showed that the mRNA and protein levels of PXR and MRP3 were markedly higher in colon cancer tissues than in non-neoplastic tissues. MRP3 mRNA was significantly correlated with PXR mRNA in cancerous and non-neoplastic colon tissues. Furthermore, the protein level of MRP3 decreased following stable RNA interference against PXR. The authors also observed that 
PXR was able to enhance or reduce cell resistance to chemotherapeutic agents when activated by rifampicin (RIF) or knocked down via short hairpin RNAs (shRNA), respectively. The results suggest that PXR may be important in human colon cancer resistance to chemotherapeutics.

Harmsen et al (51) showed that tamoxifen, vincristine, vinblastine, flutamide, ifosfamide, docetaxel and paclitaxel were able to activate PXR-mediated P-gp induction and were also shown to affect the intracellular accumulation of the P-gp probe rhodamine 123. Moreover, PXR activation was also shown to reduce the cytotoxic activity of the P-gp substrate doxorubicin in colon cancer cells. The results indicated that several anticancer drugs are able to activate PXR-mediated induction of $\mathrm{P}-\mathrm{gp}$ and affect the accumulation of $\mathrm{P}-\mathrm{gp}$ substrates. Habano et al (52) demonstrated that PXR promoter methylation was involved in the regulation of intestinal PXR and CYP3A4 mRNA expression, which may be associated with the inter-individual variability in the drug responses of colon cancer cells.

Raynal et al (53) investigated whether PXR was markedly expressed in colon tumor samples and showed a great variability of expression. The expression of hPXR in human colorectal cancer cells led to a marked chemoresistance to SN38, the active metabolite of the anticancer drug irinotecan. This result demonstrated that PXR affected the tumoral metabolism of SN38 and suggested the potential therapeutic importance of PXR quantification in the prediction of the response to irinotecan. Basseville et al (54) reported that endogenous PXR is activated in response to SN38 in human colon cancer cell lines. The authors observed that endogenous PXR translocates into the nucleus and associates with RXR upon SN38 treatment. Using ChIP, the authors demonstrated that activated endogenous PXR binds to the native promoter of the CYP3A4 gene to induce expression. RNA interference experiments supported PXR's involvement in CYP3A4 overexpression and allowed the identification of CYP3A5 and MRP2 transporter as PXR target genes. As a result, the authors observed that cells overexpressing PXR are less sensitive to irinotecan treatment. These results suggest that the PXR pathway is implicated in irinotecan resistance in a colon cancer cell line via the upregulation of specific detoxification genes.

Zheng et al (55) observed that the induction of CYP3A4 in human colon adenocarcinoma LS180 cells by hPXR agonist RIF led to a greater metabolic clearance of $1 \alpha, 25$-dihydroxyvitamin $\mathrm{D}(3)[1 \alpha, 25(\mathrm{OH})(2) \mathrm{D}(3)]$ and reduced the effects of the hormone on intestinal calcium absorption, which may contribute to an increased risk of drug-induced osteoporosis in patients receiving long-term therapy with hPXR agonists.

In addition, PXR has also been regarded as a regulator of the growth and apoptosis of colon tumors. Wang et al (56) used a xenograft model of colon cancer to define a molecular mechanism which may underlie PXR-driven colon tumor growth and malignancy. The activation of PXR was able to sufficiently enhance the neoplastic characteristics of human colon tumor cell lines and primary human colon cancer tissues xenografted into immunodeficient mice, including cell growth, invasion and metastasis. The authors also revealed that the PXR-mediated phenotype required FGF19 signaling. PXR bound to the FGF19 promoter in 'normal' intestinal crypt cells and human colon tumor cells. However, while the two cell types proliferated in response to PXR ligands, the FGF19 promoter was activated by PXR only in cancer cells. These data may lead to improved therapeutic regimens for colon cancer.

PXR and breast cancer. Breast cancer is the most common cancer in women worldwide (57). Studies suggest that PXR has a potential clinically relevant role in breast cancer. However, the relevant pathway or target genes of PXR in breast cancer biology and progression have not yet been fully clarified. Dotzlaw et al (40) first detected the expression of PXR mRNA in normal and human breast tumor tissues. The expression of PXR mRNA did not differ between the tumor tissues and adjacent matched normal breast tissues, although the level of PXR mRNA did vary among the breast tumors. The authors also observed a statistically significant inverse correlation between the level of PXR mRNA and estrogen receptor (ER) status, but no correlation with progesterone receptor (PR) status. These data indicate the possibility that PXR has a potential role in human breast tissues. Conde et al (58) processed breast tissue samples from 99 patients, including in situ, infiltrative carcinomas and benign breast diseases, by immunohistochemistry and western blot analysis. The results showed that cancer cells from patients who developed recurrent tumors exhibited high cytoplasmic levels of hPXR isoform 1 and isoform 2, while infiltrative carcinomas that recurred showed a nuclear localization for hPXR and RXR- $\alpha$. Therefore, the overexpression and the subcellular location changes of hPXR may be regarded as a potential new prognostic indicator.

Miki et al (41) detected PXR in carcinoma tissues but not in the non-neoplastic and stromal cells of breast cancers. A marked positive correlation was detected between the PXR labeling index and the histological grade and lymph node status of the carcinoma cases. PXR expression was also positively correlated with the expression of the cell proliferation marker Ki-67 in ER-positive cases. Microarray analysis showed that organic anion transporting polypeptide-A (OATP-A) was closely correlated with PXR gene expression and OATP-A mRNA and protein levels were significantly associated with PXR in breast carcinoma tissues and derived cell lines. Meyer zu Schwabedissen et al (59) also observed that the mRNA expression of OATP-1A2, a transporter capable of mediating the cellular uptake of estrogen metabolites, was $\sim 10$-fold higher in breast cancer relative to adjacent healthy breast tissues. Of note, treatment of breast cancer cells in vitro with the PXR agonist RIF-induced OATP1A2 expression in a concentration-dependent and time-dependent manner. The RIF response was abrogated following small interfering RNA (siRNA) targeting of PXR. The authors used a novel potent and specific antagonist of PXR (A-792611) to show the reversal of the RIF effect on the cellular uptake of estrone 1-sulfate (E1S), an estrogen metabolite. The data indicate that PXR and its target gene may be key to the biology of human breast cancers and may also prove to be previously unrecognized targets for breast cancer treatment.

Sandanaraj et al (60) showed that PXR*1B was associated with reduced hepatic mRNA expression of PXR and its target genes, CYP3A4 and ABCB1. Genotype-phenotype 
correlations in breast cancer patients showed PXR*1B to be significantly associated with lower doxorubicin clearance, suggesting that PXR haplotype constitution may be important in influencing interindividual and interethnic variations in the effects of its putative drug substrates.

Choi et al (61) noted that TAM-resistant MCF-7 (TAMR-MCF-7) cells expressed higher levels of MRP2 than control MCF-7 cells. Molecular analyses using MRP2 gene promoters supported the involvement of PXR in MRP2 overexpression in TAMR-MCF-7 cells. Chen et al (62) also demonstrated hPXR expression in breast cancer cell lines and normal and cancerous human breast tissue specimens. Preactivation of hPXR by SR12813 in MCF-7 cells led to an increased resistance to tamoxifen. A significant increase in resistance to Taxol was also observed in MDA-MB-231 cells with hPXR preactivation. Following activation of hPXR, the expression of MDR1 and CYP3A4, two possible mediators of hPXR-mediated drug resistance in breast cancers was increased. Furthermore, a knock-down of hPXR through shRNA sensitized MCF-7 and MDA-MB-231 cells to treatment with tamoxifen, Taxol or vinblastine. Together, the data suggest a potential role for hPXR in breast cancer resistance to drug treatment.

PXR and gynecological oncology. Masuyama et al (44) revealed various levels of PXR expression in endometrial cancer tissues but not normal tissues. Tissues showing high PXR expression exhibited markedly high expression of CYP3A4/7 and low expression of ER. HEC-1 cells, an endometrial cancer cell line, which express high PXR and low ER and PR, showed stronger transcriptional activity of the PXR-CYP3A pathway to the PXR ligands than Ishikawa cells. These results suggest that the steroid/xenobiotic metabolism may be important in the tumor tissue through PXR-CYP3A pathway, particularly in the alternative pathway for sex hormone and endocrine-disrupting chemical effects on endometrial cancer expressing low ER- $\alpha$. The authors also examined whether endocrine-disrupting chemicals (EDCs) and anticancer agents were PXR ligands. PXR-mediated transcription was markedly activated by certain steroids/EDCs through the CYP3A4-responsive element compared with the MDR1-responsive element, whereas these steroids/EDCs also enhanced the expression of CYP3A4 compared with the expression of MDR1. However, anticancer agents, including cisplatin (CDDP) and paclitaxel, were able to markedly activate PXR-mediated transcription through the MDR1-responsive element compared with the CYP3A4-responsive element, whereas these drugs were also able to enhance the MDR1 expression compared with the CYP3A4 expression (63).

Gupta et al (46) studied the presence of PXR and its effects on ovarian cancer cells following activation by its cognate ligand. In SKOV-3 cells, an ovarian carcinoma cell line, the activation of PXR by cognate ligands induces target genes (CYP2B6, CYP3A4 and UGT1A1) but not MDR1 and MRP2. PXR activation also induced SKOV-3 cell proliferation and drug resistance. In mice with SKOV-3 xenografts, RIF, a PXR agonist, induced cancer cell proliferation and tumor growth. These data served as the basis for identifying novel inhibitors of PXR activation as an approach for controlling tumor growth and preventing induction of drug resistance.
Takami et al (64) used the CDDP-sensitive Ishikawa cell line and its CDDP-resistant sub-clone (ISIW ${ }^{+}$). ISIW ${ }^{+}$cells showed higher PXR expression. When Ishikawa cells were cultured with PXR anti-sense oligonucleotides (AS), the cells did not gain CDDP resistance. In SCID mice, the authors observed that all AS-treated mice survived, whereas the controls had $50 \%$ survival at 35 days. The data indicated that PXR is a key factor for inducing, maintaining and reversing a CDDP-resistant phenotype in endometrial cancer cells. Yue et al (65) observed the expression of PXR and evaluated its clinical significance in human epithelial ovarian carcinoma. PXR was detected in 35 of 141 (24.8\%) tumor tissues and showed significant differences with age, histology, grade, ER- $\alpha$ and PR. There was a statistically significant negative correlation between the PXR expression status and disease-free survival and overall survival. The results indicate an association of PXR with ER- $\alpha$ and PR in epithelial ovarian cancers. The data support PXR as a potential prognostic factor in epithelial ovarian cancer and PXR may serve as a useful marker for identifying patients at risk of recurrence or mortality.

PXR and prostate cancer. Chen et al (47) first detected the expression of PXR in normal and cancerous prostate tissues. Pretreatment with SR12813 enhanced the resistance of PC-3 cells to Taxol and vinblastine. Futhermore, the PXR gene was knocked down, with PXR-targeting shRNA. The activity of PXR towards the promoter of CYP3A4 in PXR-ablated clones decreased compared with wild-type PC-3 cells. The cells' sensitivities to Taxol and vinblastine were increased by PXR ablation. The data indicated that PXR may be important in prostate cancer resistance to chemotherapeutic agents.

Zhang et al (66) reported a novel PXR-mediated and metabolism-based mechanism for reducing androgenic tone. The study showed that genetic or pharmacological activation of PXR reduced the androgenic activity and inhibited androgen-dependent prostate regeneration in castrated male mice receiving daily injections of testosterone propionate by inducing the expression of hydroxysteroid SULT2A1 and CYP3As, which are enzymes significant for the metabolic deactivation of androgens. In human prostate cancer cells, treatment with the PXR agonist RIF inhibited androgen-dependent proliferation of LAPC-4 cells but had little effect on androgen-independent isogenic LA99 cells. Downregulation of PXR or SULT2A1 in LAPC-4 cells by siRNA abrogated the RIF effect, indicating that the inhibitory effect of RIF on androgens was PXR- and SULT2A1-dependent. In summary, PXR may be a potential therapeutic target for lowering androgen activity and may contribute to the treatment and prevention of hormone-dependent prostate cancer.

Fujimura et al (67) investigated PXR expression in human prostate tissues. The authors identified PXR immunoreactivity using an anti-PXR antibody in benign $(n=78)$ and cancerous $(n=106)$ tissues obtained through radical prostatectomy. The authors analyzed the associations between the clinicopathological features of the patients, PXR status and CYP3A4 immunoreactivity. The experimental results showed differential PXR expression in human prostate tissues. High expression of PXR and CYP3A4 was a significant prognostic 
indicator of favorable outcomes in prostate cancer and may serve as a therapeutic target.

PXR and liver cancer. Maruyama et al (68) showed that PXR mRNA was expressed in HepG2 cells, but not human fetal liver (HFL) cells.

To examine the role of PXR in hepatocellular carcinoma (HCC) as a receptor activated by vitamin K2, Azuma et al (69) established the cells stably overexpressing PXR using an HCC cell line, HuH7. Overexpression of PXR led to reduced proliferation and motility of the cells. More marked inhibition of cellular proliferation and motility was observed when PXR overexpressing clones were treated with vitamin K2. The data indicate that the activation of PXR may contribute to the tumor suppressing effects of vitamin K2 on HCC cells. The GADD $45 \beta$ gene is a direct target of PXR and stimulates cell signals to regulate various cellular functions. Kodama and Negishi (70) demonstrated that PXR activated the GADD45 $\beta$ gene, increased p38 MAPK phosphorylation and caused HepG2 cells to change morphology and migrate.

PXR and esophageal cancer. Takeyama et al (48) performed immunohistochemical and quantitative RT-PCR evaluations in human esophageal squamous cell carcinoma (ESCC) in order to clarify the biological and clinical significance of PXR. The authors first immunolocalized PXR in 73 human ESCC cases. PXR immunoreactivity was detected in the cytoplasm and nuclei of carcinoma cells (20 and 98\% of cases, respectively). The level of nuclear PXR immunoreactivity was inversely correlated with the histological grade, lymph node metastasis status, Ki-67/MIB1 labeling index and positively correlated with the RXR- $\alpha$ status. Furthermore, multivariate analysis further demonstrated that the PXR status in carcinoma cells was able to serve as an independent favorable prognostic indicator of the patients. The results of quantitative RT-PCR showed that PXR mRNA expression was detected in $60 \%$ of cases and was notably higher in the cancerous tissues compared with the non-neoplastic tissues of the patients. This was the first study to detect the status of PXR in human ESCC and the data indicate that PXR is a significant favorable prognostic factor of human ESCC.

van de Winkel et al (71) demonstrated PXR expression in Barrett's esophagus (BE) and esophageal adenocarcinoma tissue and showed its nuclear localization in adenocarcinoma tissue. Upon activation with lithocholic acid, PXR translocated to the nuclei of OE19 adenocarcinoma cells. Together with the observed association of a PXR polymorphism and BE, the data suggest that PXR may have a potential role in the prediction and treatment of esophageal disease. The authors also revealed that PXR was able to separate high-grade dysplasia (HGD) from low-grade dysplasia (LGD) and no dysplasia (ND). PXR also appears to have diagnostic and prognostic value, but future prospective studies are required to investigate its predictive ability for neoplastic progression in BE (72).

PXR and leukemia. Kawai et al (73) first identified the expression of PXR in HL-60 human promyelocytic leukemic cells in 2003.

All-trans-retinoic acid (ATRA) is an effective treatment for acute promyelocytic leukemia and several solid tumors, but its function is limited by resistance caused by increased metabolism. Wang et al (74) designed a study to demonstrate the role of PXR in ATRA metabolism. The study indicated that the coadministration of PXR ligands was able to increase the ATRA metabolism through the activation of the PXR-CYP3A pathway, which may be a mechanism for the ATRA resistance. Other PXR target transporters may also be implicated.

PXR and osteosarcoma. Mensah-Osman et al (42) observed differences in the molecular size of the PXR protein expressed in sarcoma cell lines and the wild-type PXR expressed in the normal liver, small intestine or kidney. A polyclonal PXR antibody raised against the $\mathrm{N}$-terminus of the wild-type PXR did not detect PXR expressed in the OS187, WOL and COL osteosarcoma cell lines. In these osteosarcoma cell lines, etoposide and doxorubicin were better inducers of P450 3A4 and MDR1 compared with RIF. siRNA against PXR downregulated $\mathrm{P} 450$ 3A4 expression levels only in the osteosarcoma cell line. Cytotoxicity assays indicated that the resistance of the osteosarcoma cell lines to etoposide correlated with the PXR protein expression levels and activation of P450 3A4 and was suppressed by ketoconazole. The results suggest that PXR is important in the regulation of $\mathrm{P} 4503 \mathrm{~A} 4$ expression in osteosarcoma and its expression and activation may influence the effects of chemotherapeutic agents which induce PXR target genes implicated in drug resistance.

PXR expression is low or nonexistent in the lung, stomach, pancreas, kidneys, brain and other organs and there have been few studies of the correlation between PXR and these organic tumors at present.

\section{PXR and tumor cell apoptosis}

Studies have demonstrated that PXR is implicated in the apoptosis of tumor cells and may be an important factor in MDR.

In 2005, Zucchini et al (75) demonstrated that PXR expression was required for $\mathrm{Bcl}-2$ and $\mathrm{Bcl}-\mathrm{xL}$ upregulation upon PXR activator treatment in human and rat hepatocytes. PXR may protect the liver against harmful chemicals by simultaneously regulating detoxification and the cell apoptotic pathway.

Wang et al (76) examined the role of PXR in lipopolysaccharide (LPS)/D-galactosamine (GalN)-induced acute liver injury using PXR-null and wild-type mice. LPS/GalN-treated PXR-null mice had more marked increases in alanine transaminase (ALT), hepatocyte apoptosis, necrosis and hemorrhagic liver injury compared with wild-type mice. LPS/GalN-mediated phosphorylation of JNK1/2 and ERK1/2 was differentially regulated in PXR-null and wild-type mice. In addition, LPS/GalN-induced hepatic Stat3 survival signaling was impaired and early activation of Jak2 was delayed in PXR-null mice. After LPS/GalN treatment, the expression levels of the pro-survival proteins heme oxygenase-1 (HO-1) and Bcl-xL, which are downstream of Stat3, were markedly lower in PXR-null compared with wild-type mouse livers. The lack of PXR resulted in a significant reduction of LC3B-I and -II as well as Beclin-1 protein levels following LPS/GalN treatment. PXR is also implicated in hepatocyte homeostasis. Taken together, the results indicate that PXR is a key hepatoprotective factor. 
Masuyama et al (45) demonstrated that PXR overexpression led to a marked decrease in endometrial cancer cell growth inhibition and inhibited apoptosis in the presence of CDDP or paclitaxel. The data implied that PXR downregulation may be a novel therapeutic approach for the augmentation of sensitivity to anticancer agents or even to overcome resistance to them, in the treatment of endometrial cancer.

In a previous study, SuperArray analysis showed that PXR-mediated deoxycholic acid resistance was associated with the upregulation of multiple anti-apoptotic genes, including BIRC2, BAG3 and MCL-1 and downregulation of proapoptotic genes, such as TP53/p53 and BAK1 in human colon cancer cells (43).

However, a number of studies produced the opposite results. Ouyang et al (77) showed that PXR suppressed the proliferation and tumorigenicity of colon cancer cells by controlling the cell cycle at the $\mathrm{G}_{0} / \mathrm{G}_{1}$ phase by regulating the $\mathrm{E} 2 \mathrm{~F} / \mathrm{Rb}$ and p21 (WAF1/CIP1) pathways. Verma et al (78) also observed that the activation of PXR was antiproliferative in p53 wildtype breast cancer cells and this effect was mechanistically dependent upon the local production of $\mathrm{NO}$ and NO-dependent upregulation of p53. This finding revealed a novel biological function of PXR and suggested that a subset of PXR activators may serve as effective therapeutic and chemo-preventative agents for certain types of breast cancers. Liu et al (79) also investigated whether Tanshinone IIA (Tan IIA) has significant growth inhibition effects on U-937 cells (a human leukemic monocyte lymphoma cell line) through the induction of apoptosis. Tan IIA-induced apoptosis may result from the activation of PXR, which inhibits the activity of NF-kB and leads to the downregulation of monocyte chemoattractant protein (MCP)-1 (MCP-1/CCL2) expression.

\section{Preventing drug resistance by regulating PXR}

The hypothesis that downregulating PXR in PXR-positive cancers increases the sensitivity of cancer cells to chemotherapeutic agents has been proposed and investigated in several studies. As previously mentioned, Masuyama et al (44) showed that the downregulation of PXR by siRNA in the endometrial cancer cell line HEC-1 decreased the expression of MDR1 and sensitized cells to the anticancer agents paclitaxel and CDDP. Chen et al (61) showed that treatment with the PXR agonist SR12813 activated PXR in breast cancer cell lines and increased the expression of MDR1 and CYP3A4 and the resistance of cells to paclitaxel, vinblastine and tamoxifen. By contrast, the targeted knockdown of PXR using shRNA enhanced the sensitivity of the cells to the anticancer drugs.

One approach to overcoming PXR activation is to chemically modify the lead compound and remove its PXR-activating function without compromising the target activity. Several studies have shown this concept to be possible in principle. For example, docetaxel and paclitaxel, two inhibitors of microtubule disassembly, have minor structural differences and equal potencies in inhibiting microtubule depolymerization and cancer cell proliferation. However, paclitaxel, but not docetaxel, significantly activates PXR and regulates MDR1 expression (25). Zimmermann et al (80) reported that chemical modifications to the first generation IGF-1R inhibi- tors reduce PXR transactivation while maintaining potency against IGF-1R. However, as a result of the agonist promiscuity of PXR, an extremely large amount of effort is required in drug development programs to remove the PXR activity while maintaining the target activity for the lead compounds.

PXR antagonists that cause PXR inhibition have been demonstrated to competitively bind to PXR using in vitro binding assays. Ecteinascidin-743 (ET-743), an antineoplastic agent, has been demonstrated to suppress PXR transactivation (25). A-792611, a HIV protease inhibitor, inhibits PXR-mediated CYP3A4 expression (81). Ketoconazole, an inhibitor of CYP3A4 enzyme activity, is able to inhibit a number of NRs, including PXR, by disrupting the NR-coactivator interaction (82). Sulforaphane (SFN), an inhibitor of histone deacetylases and an inducer of phase II DMEs, shows PXR antagonist activity (83). SFN downregulates CYP3A4 expression by directly binding to PXR and inhibiting coactivator recruitment. Raynal et al showed that the activation of PXR reduced the chemosensitivity of colorectal cancer cells to irinotecan. Notably, the reduction in chemosensitivity could be reversed by SFN (53).

\section{Conclusions and prospects}

Overall, the expression of PXR is high in numerous types of tumors. Studies have demonstrated that PXR has a significant role in the drug resistance, proliferation, apoptosis and invasion of PXR-positive tumor cells. Therefore, PXR may be treated as a potentially key target in comprehensive cancer treatment and the prevention of drug resistance by regulating PXR expression is a novel and effective approach for oncotherapy.

PXR was discovered relatively recently and its structure and function have not yet been fully clarified. The correlation between PXR and multidrug resistance of tumors requires further study.

\section{References}

1. Lehmann JM, McKee DD, Watson MA, et al: The human orphan nuclear receptor PXR is activated by compounds that regulate CYP3A4 gene expression and cause drug interactions. J Clin Invest 102: 1016-1023, 1998.

2. Bertilsson G, Heidrich J, Svensson K, et al: Identification of a human nuclear receptor defines a new signaling pathway for CYP3A induction. Proc Natl Acad Sci USA 95: 12208-12213, 1998.

3. Kliewer SA, Moore JT, Wade L, et al: An orphan nuclear receptor activated by pregnanes defines a novel steroid signaling pathway. Cell 92: 73-82, 1998.

4. Blumberg B, Sabbagh W Jr, Juguilon H, et al: SXR, a novel steroid and xenobiotic-sensing nuclear receptor. Genes Dev 12: 3195-3205, 1998

5. Kast HR, Goodwin B, Tarr PT, et al: Regulation of multidrug resistance-associated protein $2(\mathrm{ABCC} 2)$ by the nuclear receptors pregnane $\mathrm{X}$ receptor, farnesoid X-activated receptor, and constitutive androstane receptor. J Biol Chem 277: 2908-2915, 2002.

6. Geick A, Eichelbaum M and Burk O: Nuclear receptor response elements mediate induction of intestinal MDR1 by rifampin. J Biol Chem 276: 14581-14587, 2001.

7. Goodwin B, Hodgson E and Liddle C: The orphan human pregnane $\mathrm{X}$ receptor mediates the transcriptional activation of CYP3A4 by rifampicin through a distal enhancer module. Mol Pharmacol 56: 1329-1339, 1999.

8. Kawana K, Ikuta T, Kobayashi Y, Gotoh O, Takeda K and Kawajiri K: Molecular mechanism of nuclear translocation of an orphan nuclear receptor, SXR. Mol Pharmacol 63: 524-531, 2003. 
9. Zhang J, Kuehl P, Green ED, Touchman JW, et al: The human pregnane $\mathrm{X}$ receptor: genomic structure and identification and functional characterization of natural allelic variants. Pharmacogenetics 11: 555-572, 2001.

10. Renaud JP, Rochel N, Ruff M, Vivat V, Chambon P, Gronemeyer H and Moras D: Crystal structure of the RAR-gamma ligand-binding domain bound to all-trans retinoic acid. Nature 378: 681-689, 1995.

11. Xu HE, Stanley TB, Montana VG, et al: Structural basis for antagonist-mediated recruitment of nuclear co-repressors by PPARalpha. Nature 415: 813-817, 2002.

12. Nolte RT, Wisely GB, Westin S, et al: Ligand binding and co-activator assembly of the peroxisome proliferator-activated receptor-gamma. Nature 395: 137-143, 1998.

13. Watkins RE, Maglich JM, Moore LB, et al: 2.1 A crystal structure of human PXR in complex with the St. John's wort compound hyperforin. Biochemistry 42: 1430-1438, 2003.

14. Rosenfeld JM, Vargas R Jr, Xie W and Evans RM: Genetic profiling defines the xenobiotic gene network controlled by the nuclear receptor pregnane $\mathrm{X}$ receptor. Mol Endocrinol 17: 1268-1282, 2003.

15. Guengerich FP: Cytochrome P-450 3A4: regulation and role in drug metabolism. Annu Rev Pharmacol Toxicol 39: 1-17, 1999.

16. Wrighton SA, Schuetz EG, Thummel KE, Shen DD, Korzekwa KR and Watkins PB: The human CYP3A subfamily: practical considerations. Drug Metab Rev 32: 339-361, 2000.

17. Moore LB, Parks DJ, Jones SA, et al: Orphan nuclear receptors constitutive androstane receptor and pregnane $\mathrm{X}$ receptor share xenobiotic and steroid ligands. J Biol Chem 275: 15122-15127, 2000.

18. Runge-Morris M, Wu W and Kocarek TA: Regulation of rat hepatic hydroxysteroid sulfotransferase (SULT2-40/41) gene expression by glucocorticoids: evidence for a dual mechanism of transcriptional control. Mol Pharmacol 56: 1198-1206, 1999.

19. Xie W, Yeuh MF, Radominska-Pandya A, et al: Control of steroid, heme, and carcinogen metabolism by nuclear pregnane $\mathrm{X}$ receptor and constitutive androstane receptor. Proc Natl Acad Sci USA 100: 4150-4155, 2003.

20. Chen C, Staudinger JL and Klaassen CD: Nuclear receptor, pregname $\mathrm{X}$ receptor, is required for induction of UDP-glucuronosyltranferases in mouse liver by pregnenolone-16 alpha-carbonitrile. Drug Metab Dispos 31: 908-915, 2003.

21. Yueh MF, Huang YH, Hiller A, Chen S, Nguyen $N$ and Tukey RH: Involvement of the xenobiotic response element (XRE) in Ah receptor-mediated induction of human UDP-glucuronosyltransferase 1A1. J Biol Chem 278 15001-15006, 2003.

22. Shelby MK and Klaassen CD: Induction of rat UDP-glucuronosyltransferases in liver and duodenum by microsomal enzyme inducers that activate various transcriptional pathways. Drug Metab Dispos 34: 1772-1778, 2006.

23. Vyhlidal CA, Rogan PK and Leeder JS: Development and refinement of pregnane $X$ receptor (PXR) DNA binding site model using information theory: insights into PXR-mediated gene regulation. J Biol Chem 279: 46779-46786, 2004.

24. Buckley DB and Klaassen CD: Induction of mouse UDP-glucuronosyltransferase mRNA expression in liver and intestine by activators of aryl-hydrocarbon receptor, constitutive androstane receptor, pregnane $\mathrm{X}$ receptor, peroxisome proliferator-activated receptor alpha, and nuclear factor erythroid 2-related factor 2. Drug Metab Dispos 37: 847-856, 2009

25. Synold TW, Dussault I and Forman BM: The orphan nuclear receptor SXR coordinately regulates drug metabolism and efflux. Nat Med 7: 584-590, 2001

26. Kast HR, Goodwin B, Tarr PT, et al: Regulation of multidrug resistance-associated protein 2 (ABCC2) by the nuclear receptors pregnane $\mathrm{X}$ receptor, farnesoid $\mathrm{X}$-activated receptor, and constitutive androstane receptor. J Biol Chem 277: 2908-2915, 2002.

27. Jones SA, Moore LB, Shenk JL, et al: The pregnane X receptor: a promiscuous xenobiotic receptor that has diverged during evolution. Mol Endocrinol 14: 27-39, 2000

28. Wentworth JM, Agostini M, Love J, Schwabe JW and Chatterjee VK: St John's wort, a herbal antidepressant, activates the steroid X receptor. J Endocrinol 166: R11-R16, 2000.

29. Savas U, Wester MR, Griffin KJ and Johnson EF: Rabbit pregnane $\mathrm{X}$ receptor is activated by rifampicin. Drug Metab Dispos 28: 529-537, 2000.

30. Zhang H, LeCulyse E, Liu L, Hu M, Matoney L, Zhu W and Yan B: Rat pregnane X receptor: molecular cloning, tissue distribution, and xenobiotic regulation. Arch Biochem Biophys 368: 14-22, 1999.
31. Cheng X and Klaassen CD: Regulation of mRNA expression of xenobiotic transporters by the pregnane $\mathrm{X}$ receptor in mouse liver, kidney, and intestine. Drug Metab Dispos 34: 1863-1867, 2006.

32. Bauer B, Hartz AM, Fricker G and Miller DS: Pregnane X receptor up-regulation of P-glycoprotein expression and transport function at the blood-brain barrier. Mol Pharmacol 66: 413-419, 2004.

33. Chirulli V, Longo V, Marini S, Mazzaccaro A, Fiorio R and Gervasi PG: CAR and PXR expression and inducibility of CYP2B and CYP3A activities in rat and rabbit lungs. Life Sci 76: 2535-2546, 2005

34. Staudinger JL, Goodwin B, Jones SA, et al: The nuclear receptor PXR is a lithocholic acid sensor that protects against liver toxicity. Proc Natl Acad Sci USA 98: 3369-3374, 2001

35. Masuyama H, Hiramatsu Y, Mizutani Y, Inoshita H and Kudo T: The expression of pregnane $\mathrm{X}$ receptor and its target gene, cytochrome P450 3A1, in perinatal mouse. Mol Cell Endocrinol 172: 47-56, 2001.

36. Dubrac S, Elentner A, Ebner S, Horejs-Hoeck J and Schmuth M: Modulation of $\mathrm{T}$ lymphocyte function by the pregnane $\mathrm{X}$ receptor. J Immunol 184: 2949-2957, 2010.

37. Owen A, Chandler B, Back DJ and Khoo SH: Expression of pregnane-X-receptor transcript in peripheral blood mononuclear cells and correlation with MDR1 mRNA. Antivir Ther 9: 819-821, 2004

38. Albermann N, Schmitz-Winnenthal FH, Z'graggen K, et al: Expression of the drug transporters MDR1/ABCB1, MRP1/ABCC1, MRP2/ABCC2, BCRP/ABCG2, and PXR in peripheral blood mononuclear cells and their relationship with the expression in intestine and liver. Biochem Pharmacol 70: 949-958, 2005.

39. Lamba V, Yasuda K, Lamba JK, Assem M, Davila J, Strom S and Schuetz EG: PXR (NR1I2): splice variants in human tissues, including brain, and identification of neurosteroids and nicotine as PXR activators. Toxicol Appl Pharmacol 199: 251-265, 2004.

40. Dotzlaw H, Leygue E, Watson P and Murphy LC: The human orphan receptor PXR messenger RNA is expressed in both normal and neoplastic breast tissue. Clin Cancer Res 5: 2103-2107, 1999

41. Miki Y, Suzuki T, Kitada K, et al: Expression of the steroid and xenobiotic receptor and its possible target gene, organic anion transporting polypeptide-A, in human breast carcinoma. Cancer Res 66: 535-542, 2006.

42. Mensah-Osman EJ, Thomas DG, Tabb MM, et al: Expression levels and activation of a PXR variant are directly related to drug resistance in osteosarcoma cell lines. Cancer 109: 957-965, 2007.

43. Zhou J, Liu M, Zhai Y and Xie W: The antiapoptotic role of pregnane $\mathrm{X}$ receptor in human colon cancer cells. Mol Endocrinol 22: 868-880, 2008.

44. Masuyama H, Hiramatsu Y, Kodama J and Kudo T: Expression and potential roles of pregnane $\mathrm{X}$ receptor in endometrial cancer. J Clin Endocrinol Metab 88: 4446-4454, 2003

45. Masuyama $\mathrm{H}$, Nakatsukasa $\mathrm{H}$, Takamoto $\mathrm{N}$ and Hiramatsu $\mathrm{Y}$ : Down-regulation of pregnane $\mathrm{X}$ receptor contributes to cell growth inhibition and apoptosis by anticancer agents in endometrial cancer cells. Mol Pharmacol 72: 1045-1053, 2007.

46. Gupta D, Venkatesh M, Wang H, et al: Expanding the roles for pregnane $\mathrm{X}$ receptor in cancer: proliferation and drug resistance in ovarian cancer. Clin Cancer Res 14: 5332-5340, 2008

47. Chen Y, Tang Y, Wang MT, Zeng S and Nie D: Human pregnane $\mathrm{X}$ receptor and resistance to chemotherapy in prostate cancer. Cancer Res 67: 10361-10367, 2007.

48. Takeyama D, Miki Y, Fujishima F, Suzuki T, Akahira J, Hata S, Miyata G, Satomi S and Sasano H: Steroid and xenobiotic receptor in human esophageal squamous cell carcinoma: a potent prognostic factor. Cancer Sci 101: 543-549, 2010.

49. Pfrunder A, Gutmann H, Beglinger C and Drewe J: Gene expression of CYP3A4, ABC-transporters (MDR1 and MRP1-MRP5) and hPXR in three different human colon carcinoma cell lines. J Pharm Pharmacol 55: 59-66, 2003.

50. Jiang H, Chen K, He J, Pan F, Li J, Chen J, Chen W and Liang $\mathrm{H}$ : Association of pregnane $\mathrm{X}$ receptor with multidrug resistance-related protein 3 and its role in human colon cancer chemoresistance. J Gastrointest Surg 13: 1831-1838, 2009.

51. Harmsen S, Meijerman I, Febus CL, Maas-Bakker RF, Beijnen JH and Schellens JH: PXR-mediated induction of P-glycoprotein by anticancer drugs in a human colon adenocarcinoma-derived cell line. Cancer Chemother Pharmacol 66: 765-771, 2010. 
52. Habano W, Gamo T, Terashima J, Sugai T, Otsuka K, Wakabayashi G and Ozawa S: Involvement of promoter methylation in the regulation of pregnane $\mathrm{X}$ receptor in colon cancer cells. BMC Cancer 11: 81, 2011.

53. Raynal C, Pascussi JM, Leguelinel G, et al: Pregnane X Receptor (PXR) expression in colorectal cancer cells restricts irinotecan chemosensitivity through enhanced SN-38 glucuronidation. Mol Cancer 9: 46, 2010.

54. Basseville A,Preisser L, de Carné Trécesson S, Boisdron-Celle M, Gamelin E, Coqueret $\mathrm{O}$ and Morel A: Irinotecan induces steroid and xenobiotic receptor (SXR) signaling to detoxification pathway in colon cancer cells. Mol Cancer 10: 80, 2011.

55. Zheng XE, Wang Z, Liao MZ, Lin YS, Shuhart MC, Schuetz EG and Thummel KE: Human PXR-mediated induction of intestina CYP3A4 attenuates $1 \alpha, 25$-dihydroxyvitamin $\mathrm{D}_{3}$ function in human colon adenocarcinoma LS180 cells. Biochem Pharmaco 84: 391-401, 2012.

56. Wang H, Venkatesh M, Li H, et al: Pregnane X receptor activation induces FGF19-dependent tumor aggressiveness in humans and mice. J Clin Invest 121: 3220-3232, 2011.

57. Khan F, Amatya B, Ng L, Demetrios M, Zhang NY and Turner-Stokes L: Multidisciplinary rehabilitation for follow-up of women treated for breast cancer. Cochrane Database Syst Rev 12: CD009553, 2012.

58. Conde I, Lobo MV, Zamora J, Pérez J, González FJ, Alba E, Fraile B, Paniagua R and Arenas MI: Human pregnane X receptor is expressed in breast carcinomas, potential heterodimers formation between hPXR and RXR-alpha. BMC Cancer 8: 174, 2008.

59. Meyer zu Schwabedissen HE, Tirona RG, Yip CS, Ho RH and Kim RB: Interplay between the nuclear receptor pregnane $X$ receptor and the uptake transporter organic anion transporter polypeptide $1 \mathrm{~A} 2$ selectively enhances estrogen effects in breast cancer. Cancer Res 68: 9338-9347, 2008.

60. Sandanaraj E, Lal S, Selvarajan V, Ooi LL, Wong ZW, Wong NS, Ang PC, Lee EJ and Chowbay B: PXR pharmacogenetics: association of haplotypes with hepatic CYP3A4 and ABCB1 messenger RNA expression and doxorubicin clearance in Asian breast cancer patients. Clin Cancer Res 14: 7116-7126, 2008.

61. Choi HK, Yang JW, Roh SH, Han CY and Kang KW: Induction of multidrug resistance associated protein 2 in tamoxifen-resistant breast cancer cells. Endocr Relat Cancer 14: 293-303, 2007.

62. Chen Y, Tang Y, Chen S and Nie D: Regulation of drug resistance by human pregnane $\mathrm{X}$ receptor in breast cancer. Cancer Biol Ther 8: 1265-1272, 2009.

63. Masuyama H, Suwaki N, Tateishi Y, Nakatsukasa H, Segawa T and Hiramatsu Y: The pregnane $\mathrm{X}$ receptor regulates gene expression in a ligand-and promoter-selective fashion. Mol Endocrinol 19: 1170-1180, 2005.

64. Takami N, Sakamoto H and Yamamoto T: Steroid and xenobiotic receptor (SXR) is a key system for the acquisition of cisplatin resistance in endometrial cancer cells. J Int Med Res 31: 59-68, 2003

65. Yue X, Akahira J, Utsunomiya H, et al: Steroid and Xenobiotic Receptor (SXR) as a possible prognostic marker in epithelial ovarian cancer. Pathol Int 60: 400-406, 2010.

66. Zhang B, Cheng Q, Ou Z, et al: Pregnane X receptor as a therapeutic target to inhibit androgen activity. Endocrinology 151: $5721-5729,2010$.

67. Fujimura T, Takahashi S, Urano T, et al: Clinical significance of steroid and xenobiotic receptor and its targeted gene CYP3A4 in human prostate cancer. Cancer Sci 103: 176-180, 2012.

68. Maruyama M,Matsunaga T, Harada E and Ohmori S: Comparison of basal gene expression and induction of CYP3As in HepG2 and human fetal liver cells. Biol Pharm Bull 30: 2091-2097, 2007.
69. Azuma K, Urano T, Ouchi Y and Inoue S: Vitamin K2 suppresses proliferation and motility of hepatocellular carcinoma cells by activating steroid and xenobiotic receptor. Endocr J 56: 843-849, 2009.

70. Kodama S and Negishi M: Pregnane X receptor PXR activates the GADD45beta gene, eliciting the p38 MAPK signal and cell migration. J Biol Chem 286: 3570-3578, 2011.

71. van de Winkel A, Menke V, Capello A, et al: Expression, localization and polymorphisms of the nuclear receptor PXR in Barrett's esophagus and esophageal adenocarcinoma. BMC Gastroenterol 11: 108, 2011.

72. van de Winkel A, van Zoest KP, van Dekken H, Moons LM, Kuipers EJ and van der Laan LJ: Differential expression of the nuclear receptors farnesoid $X$ receptor (FXR) and pregnane $X$ receptor (PXR) for grading dysplasia in patients with Barrett's oesophagus. Histopathology 58: 246-253, 2011.

73. Kawai M, Chen J, Cheung CY and Chang TK: Transcript profiling of cytochrome P450 genes in HL-60 human leukemic cells: upregulation of CYP1B1 by all-trans-retinoic acid. Mol Cell Biochem 248: 57-65, 2003.

74. Wang T, Ma X, Krausz KW, Idle JR and Gonzalez FJ: Role of pregnane $\mathrm{X}$ receptor in control of all-trans retinoic acid (ATRA) metabolism and its potential contribution to ATRA resistance. J Pharmacol Exp Ther 324: 674-684, 2008.

75. Zucchini N, de Sousa G, Bailly-Maitre B, Gugenheim J, Bars R, Lemaire $\mathrm{G}$ and Rahmani R: Regulation of Bcl-2 and Bcl-xL anti-apoptotic protein expression by nuclear receptor PXR in primary cultures of human and rat hepatocytes. Biochim Biophys Acta 1745: 48-58, 2005.

76. Wang K, Damjanov I and Wan YJ: The protective role of pregnane $\mathrm{X}$ receptor in lipopolysaccharide/D-galactosamine-induced acute liver injury. Lab Invest 90: 257-265, 2010.

77. Ouyang N, Ke S, Eagleton N, Xie Y, Chen G, Laffins B, Yao H, Zhou B and Tian Y: Pregnane X receptor suppresses proliferation and tumourigenicity of colon cancer cells. Br J Cancer 102: $1753-1761,2010$

78. Verma S, Tabb MM and Blumberg B: Activation of the steroid and xenobiotic receptor, SXR, induces apoptosis in breast cancer cells. BMC Cancer 9: 3, 2009.

79. Liu C, Li J, Wang L, Wu F, Huang L, Xu Y, Ye J, Xiao B, Meng F, Chen S and Yang M: Analysis of tanshinone IIA induced cellular apoptosis in leukemia cells by genome-wide expression profiling. BMC Complement Altern Med 12: 5, 2012.

80. Zimmermann K, Wittman MD, Saulnier MG, et al: SAR of PXR transactivation in benzimidazole-based IGF-1R kinase inhibitors. Bioorg Med Chem Lett 20: 1744-1748, 2010.

81. Healan-Greenberg C, Waring JF, Kempf DJ, Blomme EA, Tirona RG and Kim RB: A human immunodeficiency virus protease inhibitor is a novel functional inhibitor of human pregnane X receptor. Drug Metab Dispos 36: 500-507, 2008.

82. Huang H, Wang H, Sinz M, et al: Inhibition of drug metabolism by blocking the activation of nuclear receptors by ketoconazole. Oncogene 26: 258-268, 2007.

83. Zhou C, Poulton EJ, Grün F, Bammler TK, Blumberg B, Thummel KE and Eaton DL: The dietary isothiocyanate sulforaphane is an antagonist of the human steroid and xenobiotic nuclear receptor. Mol Pharmacol 71: 220-229, 2007.

84. Kliewer SA, Goodwin B and Willson TM: The nuclear pregnane $\mathrm{X}$ receptor: a key regulator of xenobiotic metabolism. Endocr Rev 23: 687-702, 2002. 